\title{
Löcher im Datenschutz der Krankenversicherer
}

\section{Thomas Eichenberger*}

* Der Autor ist auf Gesundheitsrecht spezialisiert und als Rechtsanwalt in Bern tätig. Zudem ist er Sekretär der Ärztegesellschaft des Kantons Bern und Geschäftsführer des Vereins der Leitenden Spitalärzte der Schweiz (VLSS)

Korrespondenz:

Dr. iur. Thomas Eichenberger Kellerhals Rechtsanwälte Postfach 6916

Kapellenstrasse 14

CH-3001 Bern

thomas.eichenberger@

kellerhalslaw.ch
Daten über die Gesundheit oder die Intimsphäre gelten als besonders schützenswert im Sinne des eidgenössischen Datenschutzgesetzes (DSG). Die Krankenversicherer bearbeiten im Rahmen der Durchführung der sozialen Krankenversicherung derartige, besonders sensible Persönlichkeitsdaten.

Die Bearbeitung von Gesundheitsdaten durch den Krankenversicherer muss entweder im gegenseitigen Einvernehmen mit der betreffenden Patientin oder dem betreffenden Patienten erfolgen, oder die Bearbeitung muss sich auf eine gesetzliche Grundlage abstützen, welche den Krankenversicherer zur Bearbeitung ermächtigt.

Das Bundesgesetz über die Krankenversicherung (KVG) ermächtigt den Leistungserbringer, dem Krankenversicherer eine detaillierte und verständliche Rechnung zuzustellen. Es besteht also eine gesetzliche Berechtigung zur Bearbeitung der Abrechnungsdaten. Lediglich im Abrechnungssystem des «tiers garant» (die Rechnung wird dem Patienten zugestellt, und von diesem an den Krankenversicherer weitergeleitet) hat die Patientin oder der Patient zudem die Möglichkeit, auch diese Daten zurückzuhalten und auf eine Rückerstattung durch den Krankenversicherer zu verzichten. Im «tiers payant» (die Rechnung wird dem Krankenversicherer zugestellt, und der Patient erhält eine Kopie) geht die Rechnung dagegen mit allen Details an den Krankenversicherer, bevor die Patientin oder der Patient diese gesehen hat. Aus datenschutzrechtlicher Sicht ist demzufolge der «tiers garant» $\mathrm{zu}$ bevorzugen. Folglich ist $\mathrm{zu}$ wünschen, dass der Gesetzgeber am «tiers garant» als dem von Gesetzes wegen geltenden Abrechnungssystem festhält.

\section{Sensible Daten:}

Verhältnismässigkeitsprinzip beachten

Eine zusätzliche Diagnose oder zusätzliche Auskünfte medizinischer Natur müssen im Gegensatz zu den erwähnten Abrechnungsdaten vom Versicherer ausdrücklich verlangt werden. Damit ist klar, dass sensible Gesundheitsdaten nicht automatisch und systematisch der Abrechnungsadministration des Krankenversicherers zuge- stellt und diesem mitunter breiten Personenkreis auch nicht uneingeschränkt zur Verfügung stehen sollen. Das Verhältnismässigkeitsprinzip verlangt, dass nur tatsächlich erforderliche und für den vorgesehenen Zweck geeignete Daten erhoben werden, wobei diesem Grundsatz bei besonders schützenswerten Daten erhöhte Bedeutung beizumessen ist.

Die datenschutzkonforme Umsetzung dieser Spielregeln setzt damit voraus, dass der für zusätzliche Diagnosen und zusätzliche Auskünfte medizinischer Natur angefragte Leistungserbringer mit oder ohne Auftrag der Patientin oder des Patienten vermehrt von der Befugnis Gebrauch macht, seine Antwort nur dem Vertrauensarzt oder der Vertrauensärztin abzugeben. Das Gesetz spricht zwar von «begründeten Fällen» und verlangt damit vernünftige Gründe, wenn die Initiative, die Antwort nur dem Vertrauensarzt $\mathrm{zu}$ geben, alleine vom Leistungserbringer ausgeht. Wann diese Voraussetzung gegeben ist, wird aber weder in der Krankenversicherungsgesetzgebung noch in der bundesgerichtlichen Rechtsprechung präzisiert (vgl. dazu BGE 131 II 415: Bundesgerichtsentscheid Nr. 1A.190/2004/ 1A.191/2004 vom 9. Mai 2005).

Sofern die Antwort nur dem Vertrauensarzt gegeben wird, sieht das Gesetz ausdrücklich vor, dass der Vertrauensarzt den zuständigen Stellen des Versicherers nur diejenigen Angaben weiterleitet, die notwendig sind, um über die Leistungspflicht zu entscheiden, die Vergütung festzusetzen oder eine Verfügung zu begründen. Entsprechend müssen sensible und sehr sensible Gesundheitsdaten im Kreis des Vertrauensärztlichen Dienstes bleiben.

\section{Vertrauensärzte unter Druck}

Die Krankenversicherer nehmen diese gesetzlichen Auflagen nicht immer so genau. Es sei auf die folgenden, in den letzten Jahren bekannt gewordenen Usanzen bei grösseren Krankenversicherern verwiesen:

- Im Beobachter wird über ein AVD-System (Anfragebewirtschaftung Vertrauensärztlicher Dienst) mit sensiblen Gesundheitsdaten der Versicherten der CSS berichtet, auf das angeb- 
lich anfänglich nur der Vertrauensärztliche Dienst Zugriff hatte, das aber jetzt quasi allen Mitarbeiterinnen und Mitarbeitern als elektronische Plattform zur Verfügung stehe.

- Die kantonalen Datenschutzbeauftragten und der eidgenössische Datenschutzbeauftragte mussten die Krankenversicherer und die Spitäler zurückpfeifen, weil diese vereinbart hatten, dass auf jeder Rechnung die Diagnose angegeben werden muss.

- Auf die Vertrauensärztinnen und auf die Vertrauensärzte, welche die erwähnten gesetzlichen Funktionen wahrnehmen wollen, wird erheblicher Druck ausgeübt. Sie dürfen ihre gesetzliche Filterfunktion nicht wahrnehmen. Wenn sie auf der Einhaltung des Datenschutzes beharren, riskieren sie die Anstellung oder ihr vertrauensärztliches Mandat.

- Besonders qualifizierte und engagierte Vertrauensärztinnen und Vertrauensärzte kündigen diese Funktion nach kurzer Zeit, weil sie die vom Krankenversicherer geforderte nicht gesetzeskonforme Umsetzung ihrer Aufgabe nicht mehr länger mit ihrer ärztlichen und ethischen Grundhaltung vereinbaren können.

Wer als Durchführungsorgan im Sinne des Gesetzes seine Pflichten, namentlich die Schweigepflicht, verletzt oder seine Stelle zum Nachteil Dritter, zum eigenen Vorteil oder zu einem unrechtmässigen Vorteil anderer missbraucht, wird gemäss Art. 92 lit. c KVG mit Gefängnis bis zu sechs Monaten oder mit Busse bestraft. Es handelt sich um ein Vergehen im Sinne des Straf- gesetzbuches. Das unbefugte Weiterleiten von Daten wird auch nach Art. 35 DSG vom Gesetzgeber unter Strafe gestellt.

Es mag sein, dass die Einhaltung des Datenschutzes grossmehrheitlich als lästige Aufgabe abgetan wird. Auch von den Patientinnen und Patienten wird der Datenschutz nicht immer als wichtig betrachtet. Trotzdem sind die gesetzlichen Bestimmungen einzuhalten.

Dies gilt insbesondere für die Krankenversicherer, welche als Bundesorgane mit der Durchführung der sozialen Krankenversicherung betraut sind. Nur weil in der Vergangenheit kein Kläger auf den Plan trat, musste sich nach unserem Kenntnisstand bis jetzt auch kein Richter mit Verstössen gegen den Datenschutz bei der Durchführung des KVG befassen. Dies kann sich in Zukunft ändern. Es bleibt also abzuwarten, wie lange die innerhalb der Gebäudehüllen der Krankenversicherer teilweise vorherrschende Narrenfreiheit i.S. Datenschutz noch weiter aufrechterhalten werden kann.

Vor diesem Hintergrund macht die Forderung von santésuisse, die Leistungserbringer sollten die Daten in Zukunft dem Krankenversicherer voraussetzungslos elektronisch liefern, erhebliche Mühe. Einer derartigen Lösung könnte nur zugestimmt werden, wenn gesichert wäre, dass die Patientinnen und Patienten ihre Verfügungsgewalt über die sensiblen Gesundheitsdaten weiter ausüben und die Krankenversicherer die Einhaltung des Datenschutzes garantieren können. 